\title{
A 71-year-old woman with an asymptomatic postoperative troponin elevation
}

\author{
Shannon M. Ruzycki MD, Rahim Kachra MD EdM, Kristin Lyons MDCM
}

Cite as: CMAJ 2019 January 7;191:E11-4. doi: 10.1503/cmaj.181085

A 71-year-old woman was assessed before undergoing a vaginal hysterectomy, bilateral salpingo-oophrectomy and pelvic lymph node dissection for stage I endometrial cancer. She had experienced a spontaneous subarachnoid hemorrhage in 2005, with no residual deficits, and had hypertension and osteoarthritis of the knee. She had no functional limitations, no signs or symptoms of heart failure, no chest pain or previous diagnosis of coronary artery disease, and no history of diabetes mellitus. Her blood pressure was well controlled with perindopril (2 mg daily). She was overweight (body mass index $28.7 \mathrm{~kg} / \mathrm{m}^{2}$ ); otherwise, results from a physical examination were normal. Her creatinine level was within normal limits.

\section{What preoperative investigations should be ordered for this patient?}
a. Electrocardiogram (ECG)
b. Measurement of preoperative brain natriuretic peptide (BNP)
c. Chest radiograph
d. No testing required before surgery

The correct answer is (d). The 2016 guideline for perioperative risk assessment from the Canadian Cardiovascular Society recommends that patients requiring urgent or semiurgent surgeries without features on history or physical examination to suggest an unstable coronary syndrome or an obstructive intracardiac lesion should undergo surgery without further testing (d). ${ }^{1}$ Patients requiring emergent procedures should undergo surgery regardless of suspected cardiac disease. ${ }^{1}$ As this patient did not have signs or symptoms of cardiac disease, and her surgery was considered semiurgent (surgery for cancer with a potential to metastasize), she did not have testing before surgery.

In patients undergoing elective surgery that requires an overnight admission to hospital, the society guideline recommends measurement of preoperative BNP (including the N-terminal fragment of proBNP or BNP) to predict cardiovascular complications in certain patients at high risk (b). ${ }^{1}$ Patients who are 65 years of age or older, those who are 45 years of age or older with a revised cardiac risk index of 1 or more, or those who are 18-44 years old who have vascular disease and a score of 1 or more are considered to be at high enough risk to warrant measurement of BNP (Figure 1). ${ }^{1}$ The revised cardiac risk index is a 6-point clinical risk score that assigns 1 point for each of coronary artery disease, congestive heart failure, cerebrovascular disease, diabetes mellitus requiring treatment with insulin, serum creatinine greater than $177 \mu \mathrm{mol} / \mathrm{L}$ or high-risk surgery ${ }^{2}$ (Box 1). Increasing scores correlate with higher postoperative risk of cardiac events and death. ${ }^{1,2}$ Patients who meet the above criteria who do not have preoperative BNP testing (including those who do not have BNP testing because they require emergent, urgent or semiurgent surgery) and those with elevated BNP levels should have postoperative troponin monitoring (Figure 1). Our patient was older than 65 years and had a score of 1 (for high-risk surgery); therefore, she qualified for postoperative troponin measurement.

Choosing Wisely Canada recommends against preoperative ECG (a) in asymptomatic patients undergoing noncardiac surgeries, based on evidence that ECGs do not improve preoperative risk stratification and often lead to additional, low-benefit testing. ${ }^{3}$ Our patient was asymptomatic and did not have a clear preoperative indication for an ECG. Choosing Wisely Canada also recommends against baseline radiographs of the chest (c) in asymptomatic patients preoperatively because of a high rate of false-positive results. ${ }^{3}$

We ordered troponin testing for our patient on the first, second and third days after surgery. On postoperative day 1 , her serum high-sensitivity troponin T was 50 (normal less than 14) ng/L, which rose to $87 \mathrm{ng} / \mathrm{L}$ and then $119 \mathrm{ng} / \mathrm{L}$ on serial assessments. Her ECG (Figure 2) and vital signs were normal. She had no chest pain or pressure, nausea, dyspnea or palpitations. A repeat physical examination showed no changes.

\section{What is the most likely diagnosis?}

a. Unstable angina

b. Non-ST-segment elevation myocardial infarction (non-STEMI)

c. Myocardial injury after noncardiac surgery

d. Pulmonary embolism

Our patient met the diagnostic criteria for myocardial injury after noncardiac surgery (c), defined by a postoperative rise in serum troponin that is not attributable to another cause such as sepsis ${ }^{4}$ (Box 2). Using the high-sensitivity troponin T assay, myocardial injury after noncardiac surgery can be diagnosed when the absolute value is greater than $65 \mathrm{ng} / \mathrm{L}$ or between 20 and $64 \mathrm{ng} / \mathrm{L}$ 


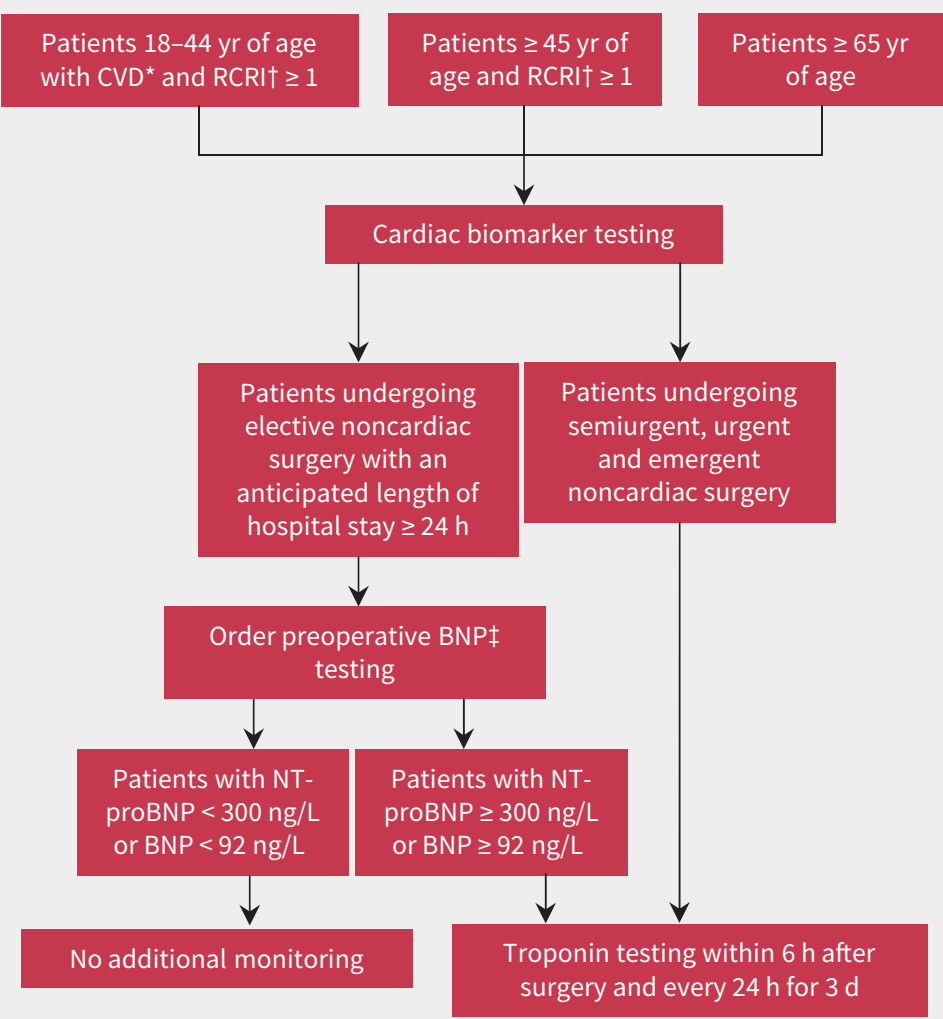

Figure 1: Canadian Cardiovascular Society recommendations for perioperative cardiac risk assessment in adult patients undergoing elective noncardiac surgery with an anticipated inpatient hospital admission longer than 24 hours. ${ }^{1}$ Note: $\mathrm{BNP}=$ brain natriuretic peptide, CVD $=$ cardiovascular disease, $\mathrm{RCRI}=$ revised cardiac risk index. *Includes coronary artery disease, cerebrovascular disease, peripheral arterial disease, congestive heart failure, pulmonary hypertension or severe obstructive intracardiac abnormality (e.g., severe aortic or mitral stenosis). †Revised cardiac risk index assigns 1 point for each of high-risk surgery (intraperitoneal, intrathoracic or superinguinal vascular surgery), coronary artery disease, congestive heart failure, cerebrovascular disease, diabetes mellitus on insulin therapy or serum creatinine level $>177 \mu \mathrm{mol} / \mathrm{L}$ (Box 1). $\ddagger$ Refers to $\mathrm{N}$-terminal (NT) fragment of proBNP or BNP.

\section{Box 1: Revised cardiac risk index criteria* and definitions ${ }^{2}$}

\section{RCRI criteria}

History of ischemic heart disease

History of congestive heart failure

History of cerebrovascular disease

Diabetes mellitus with insulin use

Preoperative serum creatinine $\geq$

$177 \mu \mathrm{mol}$

High-risk surgery

\section{Definition}

History of myocardial infarction, positive exercise stress test, current report of angina, current nitrate use or ECG with pathologic $Q$ waves

History of congestive heart failure, pulmonary edema or paroxysmal nocturnal dyspnea, or physical examination showing bilateral rales or S3 gallop, or chest radiograph showing pulmonary vascular redistribution

History of transient ischemic attack or stroke

Intraperitoneal, suprainguinal vascular or intrathoracic surgery

Note: $E C G$ = electrocardiography, RCRI = revised cardiac risk index

${ }^{*}$ The RCRI is a 6-point clinical risk score that assigns 1 point for each of the criteria listed above. ${ }^{2}$ Increasing scores correlate with higher postoperative risk of cardiac events and death. ${ }^{1,2}$

and rising by $5 \mathrm{ng} / \mathrm{L}$ on serial measurements. Myocardial injury after noncardiac surgery can also be diagnosed using a non-high-sensitivity troponin assay (Box 2). This condition is found in about $17 \%$ of patients at high risk after noncardiac surgery and is associated with a 2.7-fold increased risk of death in the first 30 days after surgery. ${ }^{4}$ Between $80 \%$ and $90 \%$ of patients with myocardial injury after noncardiac surgery are asymptomatic and are detected only with troponin surveillance. . $^{4,7}$

Although myocardial injury after noncardiac surgery includes patients with acute coronary syndromes if the diagnostic criteria for these events are also met, our patient did not have features consistent with acute coronary syndrome. Unstable angina (a) is diagnosed in patients with typical ischemic chest pain with normal troponin and ECGs. The diagnosis of nonSTEMI (b) requires ischemic symptoms or ECG changes. Pulmonary embolism (d) is an important diagnosis in the postoperative period but does not typically present with asymptomatic troponin elevation. 


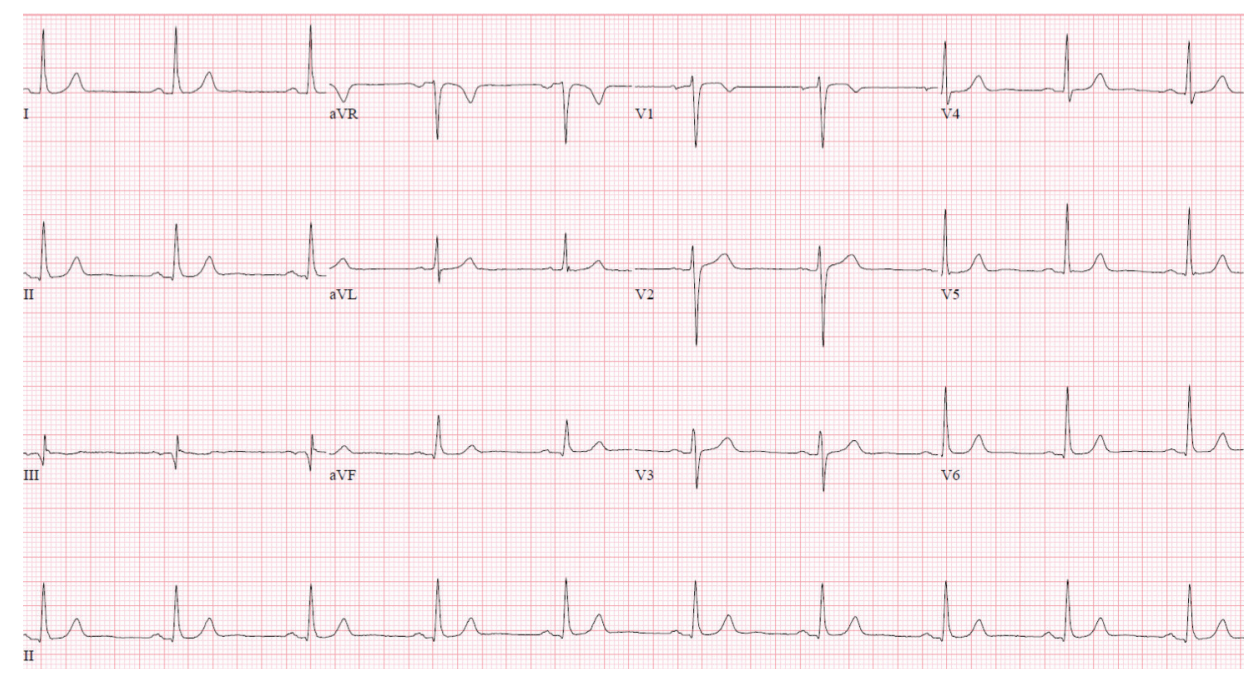

Figure 2: Postoperative electrocardiogram for a 71-year-old woman with elevated troponin, showing no features of ischemia.

\section{What is the next step in management of this patient?}

a. Coronary angiography

b. Computed tomography (CT) of the chest with pulmonary embolism protocol

c. Initiation of acetylsalicylic acid (ASA) and statin therapy with comprehensive cardiac risk assessment

d. Pharmacologic cardiac stress testing

The Canadian Cardiovascular Society guideline recommends initiation of ASA and a statin in patients with myocardial injury after noncardiac surgery (c). ${ }^{1}$ This recommendation is based on data from an international prospective cohort study involving 415 patients that showed improved survival in patients with myocardial injury after noncardiac surgery who were taking ASA and statins in the perioperative period..$^{8,9} \mathrm{~A}$ recently reported randomized clinical trial ${ }^{7}$ found that dabigatran reduced major vascular complications in patients with myocardial injury after noncardiac surgery without a significant reduction in mortality; however, these data were published after the most recent society guideline was released., ${ }^{1,7}$ To our knowledge, this latter management strategy has yet to be widely adopted.

Box 2: Troponin criteria for diagnosis of myocardial injury after noncardiac surgery ${ }^{5,6}$

\begin{tabular}{|c|c|c|}
\hline Assay & Threshold for MINS & $\begin{array}{c}\text { 30-d } \\
\text { mortality, \% }\end{array}$ \\
\hline \multirow[t]{2}{*}{$\begin{array}{l}\text { High-sensitivity } \\
\text { troponin }\end{array}$} & $\begin{array}{c}20-65 \mathrm{ng} / \mathrm{L} \text { and rising by } \\
5 \mathrm{ng} / \mathrm{L}\end{array}$ & 3.0 \\
\hline & $\geq 65 \mathrm{ng} / \mathrm{L}$ & 9.1 \\
\hline Troponin T & $\geq 0.03 \mathrm{ng} / \mathrm{mL}$ & 9.8 \\
\hline $\begin{array}{l}\text { Other troponin } \\
\text { assay }\end{array}$ & $\begin{array}{l}\text { Greater than } 99 \text { th percentile; } \\
10 \% \text { coefficient of variation }\end{array}$ & Unknown \\
\hline
\end{tabular}

Although it is probable that patients with myocardial injury after noncardiac surgery have underlying, unrecognized coronary artery disease, neither coronary angiography (a) nor stress testing (d) is currently recommended in patients with myocardial injury after noncardiac surgery in the absence of other features or symptoms. Although pulmonary embolism is an important complication in the postoperative period, CT of the chest with pulmonary embolism protocol (b) is indicated only if symptoms or signs of pulmonary embolism are present.

We prescribed atorvastatin (40 $\mathrm{mg}$ at bedtime) and ASA (81 $\mathrm{mg}$ daily). We restarted perindopril and increased the dosage to $4 \mathrm{mg}$ once daily. The patient was seen at the outpatient internal medicine follow-up clinic 30 days after discharge. Her glycated hemoglobin $A_{1 c}$ was normal. She reported episodes of palpitations, and an ECG showed frequent premature ventricular complexes. Because of these new symptoms, we ordered a myocardial perfusion imaging scan, which showed a moderate volume of ischemia in the area of the left anterior descending artery, with normal left ventricular systolic function.

Our patient underwent coronary angiography that showed a diffuse 90\% mid-left anterior descending artery lesion (Figure 3). She underwent percutaneous coronary intervention with a drugeluting stent. We prescribed dual antiplatelet therapy and referred her for cardiac rehabilitation therapy. The patient was well at six-month follow-up.

\section{Discussion}

There are nearly 500000 noncardiac surgeries performed in Canada each year. ${ }^{10}$ The mortality rate for adults undergoing elective, noncardiac surgery is $1.2 \%$, and increases in patients who have postoperative increases in serum troponin (Box 2). 4,7 Myocardial injury after noncardiac surgery, defined as an elevation of troponin level to greater than the 99th percentile of normal, is a recently recognized risk factor for death in the postoperative period. ${ }^{1}$

Management of patients at high risk in the perioperative period requires coordination of care between family physicians, 

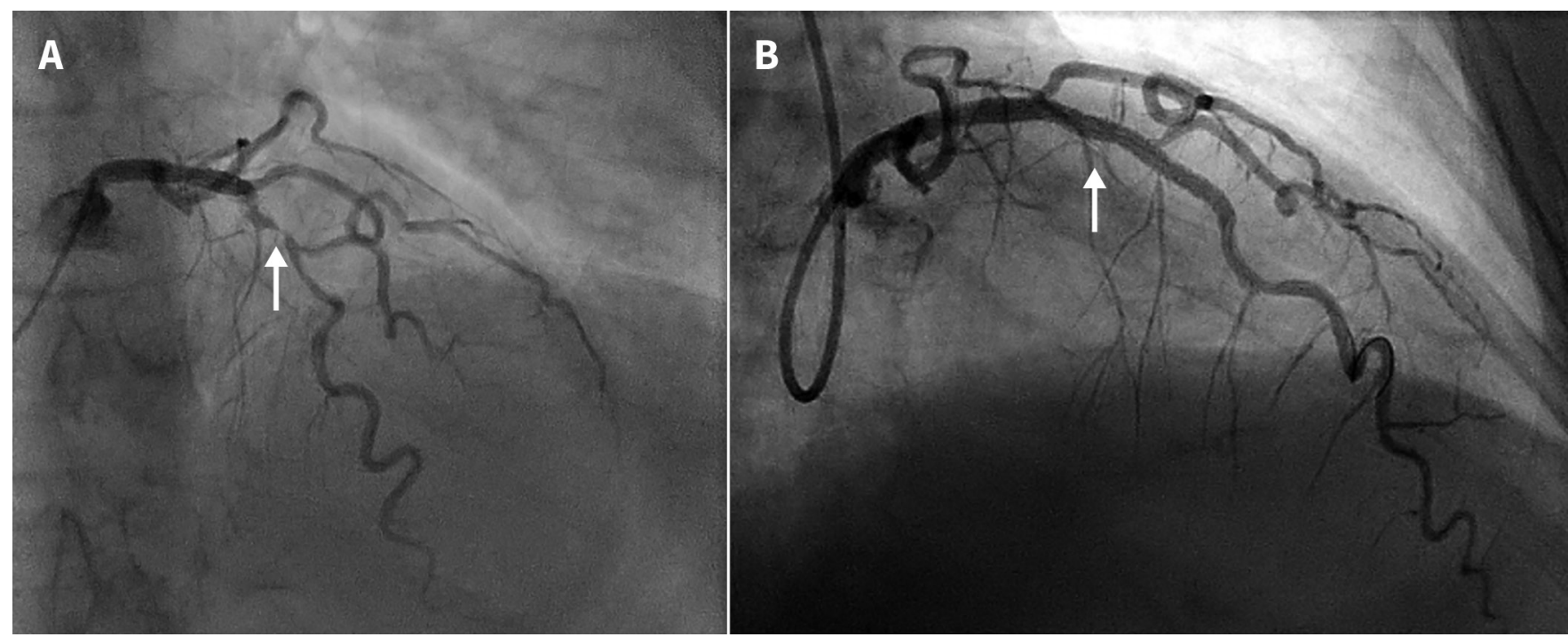

Figure 3: Cardiac angiogram for the patient showing the 90\% mid-left anterior descending artery lesion (A) before and (B) after percutaneous coronary intervention with a drug-eluting stent.

internists, anesthesiologists and surgeons. Surveillance using cardiac biomarkers is a new method for identifying patients at high-risk of perioperative complications. Patients with myocardial injury after noncardiac surgery are at high risk of short- and long-term cardiac events including death. ${ }^{4}$ Treatment begins with identifying these patients in the postoperative period using troponin screening, initiating long-term ASA and statin therapy and ensuring close clinical follow-up.

Recent evidence suggests that the use of dabigatran may have a role in reducing major vascular complications in myocardial injury after noncardiac surgery; ${ }^{7}$ however, interventions to reduce the high mortality rate observed in these patients have yet to be defined.

\section{References}

1. Duceppe E, Parlow J, MacDonald P, et al. Canadian Cardiovascular Society guidelines on perioperative cardiac risk assessment and management for patients who undergo noncardiac surgery. Can J Cardiol 2017;33:17-32.

2. Lee TH, Marcantonio ER, Mangione CM, et al. Derivation and prospective validation of a simple index for prediction of cardiac risk of major noncardiac surgery. Circulation 1999;100:1043-9.

3. Canadian Anesthesiologists' Society. Anesthesiology - Five things physicians and patients should question. Choosing Wisely Canada; 2017. Available: https://choosingwiselycanada.org/anesthesiology/ (accessed 2018 July 3).
4. Puelacher C, Lurati Buse G, Seeberger D, et al. BASEL-PMI Investigators. Perioperative myocardial injury after noncardiac surgery: incidence, mortality and characterization. Circulation 2018;137:1221-32.

5. Vascular Events in Noncardiac Surgery Patients Cohort Evaluation (VISION) Study Investigators, Devereaux PJ, Chan MT, Alonso-Coello P, et al. Association between postoperative troponin levels and 30-day mortality among patients undergoing noncardiac surgery [published erratum in JAMA 2012;307:2590]. JAMA 2012;307:2295-304.

6. Writing Committee for the VISION Study Investigators, Deveraux PJ, Biccard $\mathrm{BM}$, et al. Association of postoperative high-sensitivity troponin levels with myocardial injury and 30-day mortality among patients undergoing noncardiac surgery. JAMA 2017;317:1642-51.

7. Devereaux PJ, Duceppe E, Guyatt G, et al.; MANAGE Investigators. Dabigatran in patients with myocardial injury after non-cardiac surgery (MANAGE): an international, randomized, placebo-controlled trial. Lancet 2018;391:2325-34.

8. Berwanger O, Le Manach Y, Suzumura EA, et al. VISION Investigators. Association between pre-operative statin use and major cardiovascular complications among patients undergoing non-cardiac surgery: the VISION study. Eur Heart $J$ 2016;37:177-85.

9. Devereaux PJ, Xavier D, Pogue J, et al. POISE (PeriOperative ISchemic Evaluation) Investigators. Characteristics and short-term prognosis of perioperative myocardial infarction in patients undergoing noncardiac surgery: a cohort study. Ann Intern Med 2011;154:523-8.

10. Inpatient hospitalizations, surgeries, and newborn indicators, 2016-2017 [snap shot]. Ottawa: Canadian Institute for Health Information; 2018. Available: www.cihi.ca/en/access-data-and-reports (accessed 2018 July 3).
Competing interests: Kristin Lyons coauthored the 2016 Canadian Cardiovascular Society Guidelines on Perioperative Cardiac Risk Assessment and Management for Patients Who Undergo Noncardiac Surgery. No other competing interests were declared.

This article has been peer reviewed.

The authors have obtained patient consent.
Affiliations: Department of Medicine (Ruzycki, Kachra); W21C Research and Innovation Centre (Kachra), Cumming School of Medicine; Division of Cardiology (Lyons), Libin Cardiovascular Institute of Alberta, University of Calgary, Calgary, Alta.

Contributors: Rahim Kachra conceived the work. Kristin Lyons and Shannon Ruzycki con- ducted the literature review. All of the authors wrote the manuscript and revised it critically for important intellectual content, gave final approval of the version to be published and agreed to be accountable for all aspects of the work.

Correspondence to: Shannon Ruzycki, sarro@ualberta.ca 